\title{
Cuidados de enfermagem na prevenção de úlcera por pressão em pacientes acamados no domicílio
}

\section{Care in prevention of pressure ulcers of bedridden patients at home}

\author{
Arlene Laurenti Monterrosa Ayala ${ }^{1}$; Ana Carolina Braz Pelário da Silva Galende ${ }^{2}$; \\ Francine Renata Stoeberl ${ }^{2}$
}

\begin{abstract}
Resumo
Este estudo foi desenvolvido na área de abrangência de uma Unidade Básica de Saúde em Joinville/SC com pacientes acamados no domicílio. Teve como objetivos, caracterizar o risco dos acamados para o desenvolvimento de úlcera por pressão; descrever os fatores propícios para o seu surgimento e avaliar a adesão aos cuidados prescritos na prevenção desta lesão. Trata-se de um estudo descritivo e exploratório. A coleta de dados foi realizada por meio de instrumento contendo dados de identificação, condições clínicas dos pacientes e pela aplicação da escala de Braden. Foram avaliados 12 pacientes acamados. A média de idade dos pacientes avaliados foi de 80,5 anos. A maioria era branca, com baixa escolaridade e dependente dos serviços públicos de saúde. Todos os pacientes possuíam alguma doença de base, sendo a mais frequente o Acidente Vascular Cerebral. O tempo de acamado foi de 67 meses $( \pm 53)$. A mobilidade e a percepção sensorial estavam completamente ou muito limitadas, em $83 \%$ e $50 \%$ dos avaliados, respectivamente. $67 \%$ dos usuários apresentavam o estado nutricional muito pobre ou inadequado e $92 \%$ a pele constantemente úmida ou muito úmida. A baixa ingesta hídrica esteve presente para todos os participantes. A adesão dos cuidadores aos cuidados prescritos foi parcial. Ao final do estudo constatou-se que os achados estão em consonância com os dados da literatura e que os pacientes investigados possuíam um risco elevado para o desenvolvimento de úlcera por pressão, uma vez que os fatores propícios ao aparecimento da lesão se mostravam com frequências elevadas.
\end{abstract}

Palavras chave: Úlcera por pressão. Assistência domiciliar. Fatores de risco não é um descritor da saúde do DECS/. OBS: a palavra chave fatores de risco aparece na biblioteca virtual em saúde como um descritor em ciências da saúde.

\begin{abstract}
This study was carried out in the catchment area of a Basic Health Unit in Joinville/SC bedridden patients receiving home care. We aimed to characterize the risk of the bedridden to develop pressure ulcers, describing the factors favorable to its emergence, and evaluate adherence to treatment prescribed to prevent this injury. This is a descriptive study. Data collection was carried out through an instrument containing identification data, clinical conditions of patients and the application of the Braden scale. Twelve bedridden patients were evaluated. The average age of the patients evaluated was 80.5 years. Most were white, with low education and dependent on public health services. All patients had some underlying disease, the most frequent the Stroke (CVA). The laid up time was 67 months $( \pm 53)$. The mobility and sensory perception were completely or limited in $83 \%$ and $50 \%$ of the patients evaluated, respectively. Sixty-seven percent of users had very poor nutritional status or inadequate and $92 \%$ were constantly moist or very moisty. Low fluid intake was present for all participants. Adherence to prescribed care of the caregivers was partial. The findings at the end of the study are consistent with the literature and the studied patients had an elevated risk for developing pressure ulcers, since the factors favorable to the appearance of the lesion showed with high frequencies.
\end{abstract}

Keyword: Pressure ulcer. Home care. Risk factors.

\footnotetext{
${ }^{1}$ Enfermeira. Mestre em Saúde Pública pelo Centro de Ciências da Saúde da Universidade Federal de Santa Catarina (UFSC). Professora do Instituto Superior e Centro Educacional Luterano Bom Jesus/Ielusc, Joinville (SC). E-mail: alayala@bol.com.br

${ }^{2}$ Enfermeira pelo Instituto Superior e Centro Educacional Luterano Bom Jesus/Ielusc, Joinville (SC).
} 


\section{Introdução}

As úlceras por pressão (UPs) são consideradas um problema de saúde pública, de grande relevância para o setor de saúde, porque sua causalidade transparece a forma de cuidado que está sendo prestado a pessoa, além de serem consideradas evitáveis. Elas estão associadas à dor e sofrimento do paciente, ao aumento no tempo de internação e dos custos de saúde (ROCHA FILHO et al., 2013).

Estratégias de prevenção com alguma evidência de eficácia incluem a orientação aos pacientes e/ou cuidadores sobre a exposição a fatores de risco para o aparecimento da lesão.

Os principais fatores de risco descritos na literatura são a mobilidade prejudicada, desnutrição e baixo índice de massa corporal (IMC), problemas de saúde física, incluindo os que afetam a oxigenação e perfusão, idade avançada, temperatura corporal, fricção e cisalhamento, umidade da pele, dificuldades cognitivas e de percepção sensorial (ARAÚJO; MOREIRA; CAETANO, 2011; POSS et al., 2010).

Estudos que examinaram as intervenções para a prevenção de úlcera, verificaram que estratégias, como por exemplo, a aplicação de escalas preditivas, o uso de superfícies de apoio, mudança de decúbito, recomposição do estado nutricional e hidratação, foram adequadas na prevenção do desenvolvimento dessa lesão (BRITO; SOARES; SILVA, 2014).

O estudo de Pessoa, Rocha e Bezerra (2011) realizado em uma área de ESF, localizada na região sul de Teresina, refere que a prevalência de úlcera por pressão em acamados no domicílio foi de 13,3\%. Outro estudo, que investigou pacientes sob assistência domiciliária em um distrito de saúde de Ribeirão Preto mostrou, que a prevalência de pacientes com úlcera por pressão (UPP) foi 19,1\%, considerando que, dos 47 pacientes, nove apresentavam úlceras por pressão (CHAYAMITI; CALIRI, 2010).

Tendo em mente, as pesquisas que demonstram a incidência de úlcera por pressão em pacientes acamados, conhecer os indivíduos acamados no domicílio em risco de desenvolver a UPP na área de abrangência de uma Unidade Básica de Saúde em Joinville (SC), é de extrema relevância. A identificação de tais indivíduos pode ser útil à equipe de saúde, uma vez que cabe a ela buscar a implementação de ações que possam prevenir esse tipo de lesão. E além disso, pode possibilitar aos profissionais de saúde qualificar o cuidado em saúde que é prestado aos usuários assistidos pela UBS.

Diante disso, os objetivos desta investigação foram caracterizar os pacientes - segundo a escala de Braden - em risco à formação de úlcera por pressão (UPP), descrever os fatores propícios para o seu surgimento, e avaliar a adesão dos pacientes aos cuidados prescritos na prevenção do aparecimento de UPP.

\section{Material e Método}

Este é um estudo descritivo exploratório realizado em 2013 com pacientes acamados no domicílio e em risco de desenvolver úlcera por pressão. Foi desenvolvido na área de abrangência da Unidade Básica de Saúde (UBS) do Itaum em Joinville/SC. Para a escolha do local do estudo foi considerado as questões de logística e a receptividade dos profissionais da UBS ao estudo.

O bairro do Itaum possui um território de 3,18 $\mathrm{Km}^{2}$, sua população estimada é de 15.375 habitantes, com renda per capita de 1,77 salários mínimos por mês. Seu potencial econômico em relação a $100 \%$ do município de Joinville representa 7,8\% do potencial econômico total, dividido da seguinte forma: 2,05\% no setor industrial, 3,06\% no comércio, e 2,69\% no setor de serviços (PREFEITURA MUNICIPAL DE JOINVILLE, 2015).

Numa primeira aproximação, foram apresentados o tema do estudo, sua relevância social e seus objetivos aos profissionais da UBS. Visando conhecer e orientar a seleção da população a ser estudada, buscou-se junto aos profissionais da unidade, informações acerca dos pacientes acamados, precisamente - quantos eram e seus respectivos endereços. 
A seleção dos pacientes deu-se em duas fases: na primeira, foram selecionados e caracterizados 15 pacientes. A caracterização ocorreu durante a visita domiciliária por meio da aplicação da escala de Braden (BRADEN; BERGSTROM, 1987), sendo os pacientes categorizados em risco leve, moderado e alto para desenvolvimento de úlcera por pressão. $\mathrm{Na}$ segunda fase, foram escolhidos apenas os usuários em risco moderado e alto para o desenvolvimento de UPP e que concordaram por meio da assinatura do Termo de Consentimento Livre e Esclarecido (TCLE), em participar do estudo. Foram excluídos do estudo três usuários, dois por possuírem algum tipo de lesão e um por apresentar risco leve para o desenvolvimento de úlcera por pressão. A amostra da pesquisa foi composta, portanto, de 12 pacientes. Esta amostra justifica-se à medida que o trabalho tem por objetivo fazer um estudo exploratório da problemática. Não participaram do estudo acamados no domicílio com grau leve para o aparecimento de UPP e os que já apresentavam lesão.

Quanto à escala de Braden, ela é organizada em seis subescalas que apresentam escores variando de 01 a 04, que ao final quando somados indicam o escore geral, que pode variar de 06 a 23. Os usuários que obtiveram o escore igual ou maior que 16 foram considerados de pequeno risco para o desenvolvimento de úlceras de pressão; escores entre 11 e 16 indicaram risco moderado e com escore abaixo de 11, alto risco (BRADEN; BERGSTROM, 1987).

A avaliação dos pacientes considerou os seguintes pontos e escores:

a) Percepção sensorial: avalia a capacidade do paciente em reagir significativamente à pressão relacionada ao desconforto. Completamente limitado (um ponto): o paciente não reage a estímulo doloroso. Muito limitado (dois pontos): reage somente a estímulo doloroso, não é capaz de comunicar desconforto. Levemente limitado (três pontos): responde ao comando verbal, mas nem sempre comunica o desconforto. Nenhuma limitação (quatro pontos). b) Umidade: avalia a que nível a pele está exposta à umidade. Constantemente úmida (um ponto): a pele é mantida úmida quase constantemente por transpiração, urina, etc. Muito úmida (dois pontos). Ocasionalmente úmida (três pontos). Raramente úmida (quatro pontos).

c) Atividade: avalia o grau de atividade física do paciente. Acamado (um ponto). Restrito à cadeira (dois pontos): a capacidade de andar está severamente limitada ou nula. Caminha ocasionalmente (três pontos): anda durante o dia, mas passa a maior parte de cada turno na cama ou na cadeira. Caminha frequentemente (quatro pontos).

d) Mobilidade: avalia a capacidade de mudar e controlar a posição do corpo. Completamente imobilizado (um ponto). Muito limitado (dois pontos): faz pequenas mudanças ocasionais na posição do corpo, mas é incapaz de fazer mudanças frequentes sozinho. Levemente limitado (três pontos): faz frequente, embora pequenas, mudanças na posição do corpo sem ajuda. Nenhuma limitação (quatro pontos).

e) Nutrição: avalia o padrão usual de consumo alimentar. Muito pobre (um ponto): o paciente nunca come uma refeição completa. Ingere pouco líquido. Provavelmente inadequado (dois pontos): raramente come uma refeição completa. Ou recebe abaixo da quantidade satisfatória de dieta líquida ou alimentação por sonda. Adequada (três pontos): come mais da metade das refeições. Come um total de quatro porções de alimento rico em proteína (carne ou laticínio) diariamente. $\mathrm{Ou}$ é alimentado por sonda ou regime de nutrição Parenteral Total. Excelente (quatro pontos). Come quatro ou mais porções de carne e laticínios. Não requer suplemento alimentar.

f) Fricção e cisalhamento: avalia o grau de contato entre a pele do paciente e o lençol, de acordo com seu grau de mobilidade. Problema (um ponto): quando o paciente requer assistência de moderada à máxima para se mover. Há quase sempre atrito 
da pele com o lençol. Potencial para problema (dois pontos): move-se, mas, sem vigor. Na maior parte do tempo mantém posição boa na cama ou cadeira. Nenhum problema (três pontos): movese sozinho e tem suficiente força muscular para erguer-se completamente durante o movimento.

Após a caracterização da amostra do estudo procedeu-se nos dias subsequentes o exame físico e a observação dos pacientes em seus domicílios. Durante este processo buscou-se os dados de identificação: idade, raça, sexo e escolaridade. Dados das condições clínicas: sinais vitais, estado nutricional, mobilidade, perfusão tecidual, hidratação e condições da pele. E dados de fatores propícios ao surgimento das úlceras de pressão, como: tabagismo atual, tabagismo pregresso, tempo de acamado, doença de base, alcoolismo e outras doenças associadas (POSS et al., 2010).

Sequencialmente foram pesquisados os prontuários dos usuários na Unidade Básica de Saúde, com o intuito de buscar informações que pudessem subsidiar o processo de investigação e de prescrição de cuidados.

A prescrição de cuidados aos pacientes acamados foi norteada a partir das necessidades alteradas que os usuários apresentavam tendo como referência as necessidades básicas e as evidencias identificadas e observadas por ocasião da aplicação da Escala de Braden (BRADEN; BERGSTROM, 1987). Em continuação, foram realizadas duas visitas semanais aos usuários, no período de março a julho de 2013, para avaliar a adesão dos acamados e/ou dos cuidadores aos cuidados prescritos.

Finalizando, as informações e dados coletados foram tratados como a seguir: as variáveis quantitativas foram inseridas em uma planilha Excel, desenhada para o estudo, calculadas por meio de medidas de tendência central - média, desvio padrão e percentual - apresentados sob forma de tabelas e gráficos; e as evidências científicas presentes na literatura relacionadas aos fatores de risco para a úlcera de pressão foram comparadas às informações obtidas na coleta de dados e na aplicação da Escala de Braden
(BRADEN; BERGSTROM, 1987). Tal confrontação objetivou identificar as discordâncias com os achados ou similaridades. As conclusões orientaram a prescrição de cuidados na prevenção de UP.

$\mathrm{O}$ estudo foi submetido à apreciação do Comitê de Ética, sendo aprovado sob o protocolo 05072012.2.0000.5365.

\section{Resultados}

No presente estudo constatou-se que a média de idade dos indivíduos investigados foi de 80,5 anos $( \pm 4,8)$. Quanto à raça e sexo, a maioria dos sujeitos do estudo era pertencente à raça branca (83\%), sendo divididos de acordo com o sexo em partes iguais. Referente à escolaridade $76 \%$ dos pacientes possuíam $1^{\mathrm{o}}$ grau incompleto, $8 \% 1^{\circ}$ e $2^{\circ}$ grau completo e para o analfabetismo foi encontrado o percentual de $8 \%$. O tempo de acamado variou entre dois meses e 11 anos, sendo que a média foi de 67 meses $( \pm 53)$.

O tabagismo e o etilismo não foram evidenciados neste estudo como fatores de risco para UP, em virtude de o grupo investigado ser constituído, por pessoas não tabagistas e etilistas no momento do estudo. Entretanto, a prevalência de tabagismo e etilismo pregresso esteve presente na população alvo, sendo que $33 \%$ dos investigados haviam fumado ou utilizado bebidas alcoólicas em anos passados.

A amostra estudada em sua maioria era dependente dos serviços públicos de saúde. Observou-se que dos 12 indivíduos investigados, (25\%) utilizavam o SUS e também um plano privado de atendimento, (25\%) apenas um plano privado e os outros $(50 \%)$ utilizavam somente o SUS.

No que tange às condições de saúde, foi constatado que todos os pacientes possuíam alguma doença de base, sendo a mais frequente o Acidente Vascular Cerebral (AVC) em nove (76\%) dos usuários. Alzheimer, gangrena e fratura de fêmur foi referido por três usuários, correspondendo ao percentual de $8 \%$ de usuários para cada doença referida. Observou-se que todos os participantes apresentavam no mínimo duas comorbidades sendo 
as mais frequentes, a Hipertensão Arterial (HA), Diabetes mellitus (DM) e doença de Alzheimer
(DA), com os percentuais de $92 \%, 83 \%$ e $17 \%$ dos casos, respectivamente (TABELA 1).

Tabela 1 - Distribuição dos usuários investigados quanto à doença de base e comorbidades em 2013, Joinville/SC.

\begin{tabular}{c|c|c|c}
\hline Doença de base & Comorbidades & N & \% \\
\hline Acidente Vascular Cerebral & Hipertensão arterial/Diabetes mellitus & 7 & 60 \\
Acidente Vascular Cerebral & Hipertensão arterial & 1 & 8 \\
Acidente Vascular Cerebral & Câncer/Insuficiência renal crônica & 1 & 8 \\
Fratura de Fêmur & Hipertensão arterial/Diabetes mellitus & 1 & 8 \\
Gangrena & Hipertensão arterial/Diabetes mellitus & 1 & 8 \\
Alzheimer & Hipertensão arterial/Diabetes mellitus & 1 & 8 \\
\hline
\end{tabular}

Fonte: prontuários

Os resultados após a aplicação da Escala de de UPP, em razão dos fatores predisponentes Braden mostram que os usuários investigados aparecerem com frequência elevada, conforme o possuíam um alto risco para o desenvolvimento quadro a seguir:

Quadro 1 - Parâmetros de risco para úlcera por pressão dos usuários investigados em 2013 de acordo com a Escala de Braden, Joinville (SC).

\begin{tabular}{|c|c|c|c|c|c|c|}
\hline $\begin{array}{c}\text { Usuário/ } \\
\text { Risco para } \\
\text { UPP }\end{array}$ & $\begin{array}{c}\text { Percepção } \\
\text { sensorial }\end{array}$ & $\begin{array}{l}\text { Umidade da } \\
\text { pele }\end{array}$ & $\begin{array}{l}\text { Atividade } \\
\text { Física }\end{array}$ & $\begin{array}{c}\text { Fricção e } \\
\text { Cisalhamento }\end{array}$ & Nutrição & Mobilidade \\
\hline $\begin{array}{c}\text { A } \\
\text { Moderado }\end{array}$ & $\begin{array}{l}\text { Levemente } \\
\text { limitado }\end{array}$ & Muito Úmida & $\begin{array}{c}\text { Caminha } \\
\text { Ocasionalmente }\end{array}$ & $\begin{array}{c}\text { Potencial para } \\
\text { Problema }\end{array}$ & $\begin{array}{l}\text { Muito } \\
\text { Pobre }\end{array}$ & $\begin{array}{l}\text { Levemente } \\
\text { limitado }\end{array}$ \\
\hline $\begin{array}{c}\text { B } \\
\text { Moderado }\end{array}$ & $\begin{array}{l}\text { Levemente } \\
\text { limitado }\end{array}$ & Muito Úmida & $\begin{array}{l}\text { Restrito a } \\
\text { Cadeira }\end{array}$ & $\begin{array}{c}\text { Potencial para } \\
\text { Problema }\end{array}$ & Inadequada & $\begin{array}{l}\text { Levemente } \\
\text { limitado }\end{array}$ \\
\hline $\begin{array}{c}\text { C } \\
\text { Alto }\end{array}$ & $\begin{array}{l}\text { Levemente } \\
\text { limitado }\end{array}$ & $\begin{array}{l}\text { Constantemente } \\
\text { Úmida }\end{array}$ & Acamado & Problema & Inadequada & Muito limitado \\
\hline $\begin{array}{c}\text { D } \\
\text { Alto }\end{array}$ & $\begin{array}{l}\text { Completamente } \\
\text { Limitado }\end{array}$ & $\begin{array}{l}\text { Ocasionalmente } \\
\text { Úmida }\end{array}$ & Acamado & Problema & $\begin{array}{l}\text { Muito } \\
\text { Pobre }\end{array}$ & Muito limitado \\
\hline $\begin{array}{c}\mathrm{E} \\
\text { Alto }\end{array}$ & Muito limitado & $\begin{array}{l}\text { Constantemente } \\
\text { Úmida }\end{array}$ & Acamado & Problema & $\begin{array}{l}\text { Muito } \\
\text { pobre }\end{array}$ & Muito limitado \\
\hline $\begin{array}{c}\text { F } \\
\text { Alto }\end{array}$ & Muito Limitado & $\begin{array}{l}\text { Constantemente } \\
\text { Úmida }\end{array}$ & Acamado & Problema & $\begin{array}{l}\text { Muito } \\
\text { Pobre } \\
\text { (sonda) }\end{array}$ & $\begin{array}{l}\text { Completamente } \\
\text { Imobilizado }\end{array}$ \\
\hline$\frac{\mathrm{G}}{\text { Moderado }}$ & $\begin{array}{l}\text { Levemente } \\
\text { Limitado }\end{array}$ & Muito Úmida & $\begin{array}{l}\text { Restrito a } \\
\text { Cadeira }\end{array}$ & Problema & Adequada & Muito Limitado \\
\hline $\begin{array}{c}\mathrm{H} \\
\text { Moderado }\end{array}$ & Muito Limitado & Muito Úmida & Acamado & Problema & Adequada & Muito Limitado \\
\hline $\begin{array}{c}\text { I } \\
\text { Moderado }\end{array}$ & $\begin{array}{l}\text { Levemente } \\
\text { Limitado }\end{array}$ & Muito Úmida & $\begin{array}{l}\text { Restrito a } \\
\text { Cadeira }\end{array}$ & Problema & Adequada & Muito Limitado \\
\hline
\end{tabular}

continua... 
continuação...

\begin{tabular}{|c|c|c|c|c|c|c|}
\hline $\begin{array}{c}\text { Usuário/ } \\
\text { Risco para } \\
\text { UPP }\end{array}$ & $\begin{array}{c}\text { Percepção } \\
\text { sensorial }\end{array}$ & $\begin{array}{c}\text { Umidade da } \\
\text { pele }\end{array}$ & $\begin{array}{c}\text { Atividade } \\
\text { Física }\end{array}$ & $\begin{array}{c}\text { Fricção e } \\
\text { Cisalhamento }\end{array}$ & Nutrição & Mobilidade \\
\hline $\begin{array}{c}\mathrm{J} \\
\text { Moderado }\end{array}$ & $\begin{array}{c}\text { Levemente } \\
\text { limitado }\end{array}$ & Muito Úmida & $\begin{array}{c}\text { Restrito a } \\
\text { Cadeira }\end{array}$ & Problema & $\begin{array}{c}\text { Inadequada } \\
\text { Muito } \\
\mathrm{K}\end{array}$ & $\begin{array}{c}\text { Completamente } \\
\text { Imobilizado }\end{array}$ \\
$\begin{array}{c}\text { Alto } \\
\mathrm{L}\end{array}$ & Muito limitado & $\begin{array}{c}\text { Constantemente } \\
\text { Úmida }\end{array}$ & Acamado & Problema & Muito Limitado \\
Moderado & Muito Limitado & Muito Úmida & Acamado & Problema & Adequada & Muito limitado \\
\hline
\end{tabular}

Fonte: Prontuário /anamnese/exame clínico

Em relação à percepção sensorial, 50\% dos pacientes apresentaram muita limitação ou completa limitação.

No presente estudo, $92 \%$ dos pacientes investigados mostrou a pele constantemente úmida ou muito úmida, sendo que $50 \%$ usavam fraldas.

Em relação à mobilidade, $83 \%$ dos pacientes apresentaram-se completamente ou muito limitados. Entretanto, apesar do problema, 58\% dos participantes do estudo não utilizavam superfícies de apoio como, por exemplo, colchões piramidais.

A maior parte dos usuários $67 \%$ apresentou o estado nutricional muito pobre ou inadequado, isso devido às dificuldades com a mastigação em razão da dentição precária, e deglutição em virtude de sequela do AVC. A média de ingesta hídrica encontrada por individuo foi de $475 \mathrm{ml} / \mathrm{dia}$ ( $(150)$.

Quanto à fricção e cisalhamento $83 \%$ dos participantes tinham problema, pois necessitavam de auxílio para reposicionarem-se na cama ou cadeira, os outros $17 \%$ apresentavam problema em potencial.

$\mathrm{Na}$ sequência, é apresentada a distribuição dos usuários quanto aos escores das sub escalas (GRÁFICO 1). Neste estudo, a umidade da pele, atividade física, fricção e cisalhamento foram os itens que obtiveram os menores escores.

Gráfico 1 - Frequências relativas das pontuações (1 a 4) das subescalas de Braden nos pacientes estudados em 2013 em Joinville (SC).

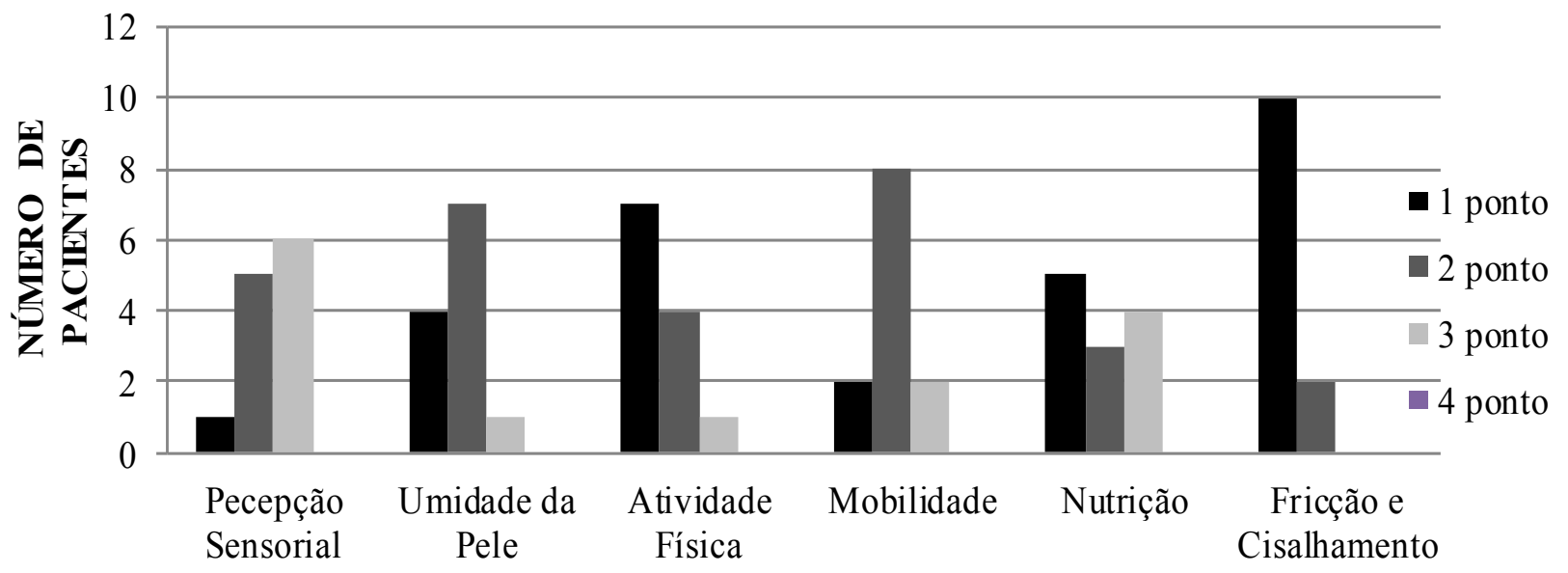

Fonte: anamnese/exame clínico 
Após a análise atenta dos dados de cada paciente, obtidos por meio da aplicação da Escala de Braden (BRADEN; BERGSTROM, 1987) e do exame foi elaborada a prescrição de enfermagem de forma individualizada. Tal prescrição teve por objetivo orientar os usuários e cuidadores para alguns cuidados na prevenção de UPP que não eram realizados, ou que eram realizados, mas necessitavam ser aprimorados ou reforçados. Dentre os cuidados $80 \%$ estavam relacionados à etiologia da úlcera por pressão. Destes, $6 \%$ diziam respeito à percepção sensorial prejudicada, $31 \%$ à nutrição comprometida, $32 \%$ à umidade e integridade da pele prejudicada, $25 \%$ relativo à mobilidade diminuída e $6 \%$ a cuidados concernentes à fricção e cisalhamento. Os demais cuidados prescritos estavam relacionados ao autocuidado e ao meio ambiente. Os cuidados prescritos em um total de 20 podem ser identificados no quadro 2 .

Quadro 2 - Distribuição dos cuidados prescritos aos usuários investigados segundo os problemas identificados na aplicação da Escala de Braden e no exame físico em 2013, Joinville (SC).

\begin{tabular}{|c|c|}
\hline 12 & Realizar inspeção e hidratação da pele \\
\hline 12 & Secar bem a pele após banho \\
\hline 12 & Observar sinais de hiperemia \\
\hline 12 & Oferecer dieta pastosa \\
\hline 12 & Esticar bem os lençóis \\
\hline 12 & Deixar objetos próximos ao alcance \\
\hline 12 & Tomar medicamentos nos horários certos \\
\hline 11 & Realizar dieta hipossódica \\
\hline 11 & Realizar dieta hipocalórica \\
\hline 10 & Orientar para aumento da ingesta hídrica \\
\hline 08 & Realizar mudança de decúbito de 04 em 04 horas \\
\hline 08 & Proteger proeminências ósseas com coxins, almofadas \\
\hline 07 & Utilizar colchão piramidal \\
\hline 06 & Realizar troca de fralda \\
\hline 04 & Observar nível de consciência \\
\hline 04 & Manter grades do leito elevadas \\
\hline 03 & Deambular com auxilio \\
\hline 03 & Esvaziar bolsa coletora de SVD \\
\hline 02 & Preparar alimentos ricos em fibras \\
\hline 01 & Deixar ambiente limpo e arejado \\
\hline
\end{tabular}

Fonte: anamnese/exame clínico 
A partir da análise dos 20 diferentes cuidados prescritos foi constatado que a média de adesão à prescrição foi de $51 \%( \pm 30)$. Os cuidados relativos à prevenção de úlcera por pressão - inspeção e hidratação da pele, secar a pele após o banho, observar sinais de hiperemia, oferecer dieta pastosa, esticar lençóis e deixar objetos ao alcance do paciente - foram prescritos para todos os pacientes. Entretanto, foi observado que a adesão aos cuidados foi de $75 \%, 25 \%, 42 \%, 83 \%$, $33 \%$ e 58\%, respectivamente (GRÁFICO 2).

Gráfico 2 - Frequência da efetividade a prescrição de enfermagem para os usuários investigados em 2013, Joinville (SC)

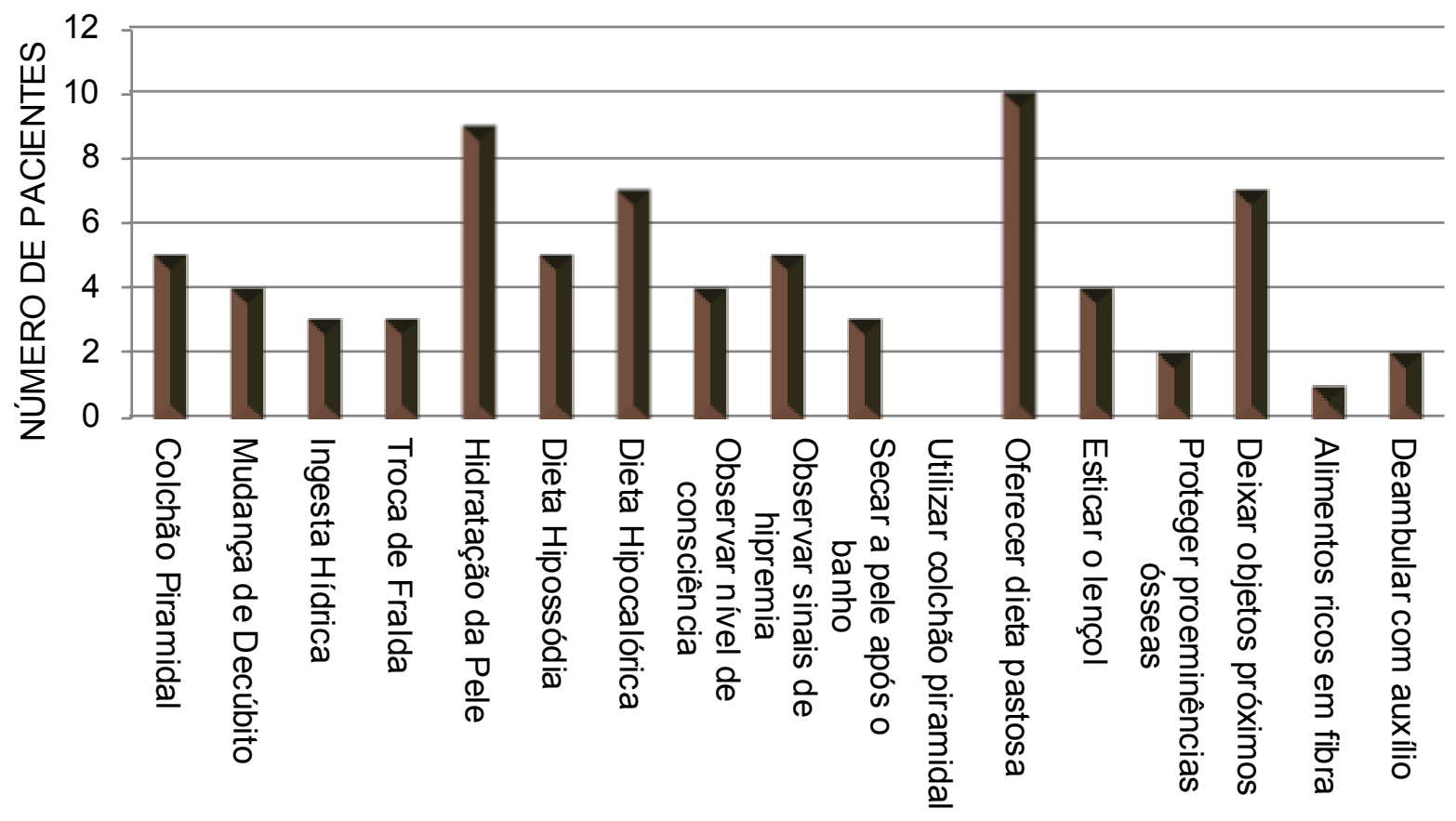

Fonte: anamnese

Dos 12 pacientes acamados cinco utilizavam o colchão piramidal. Todavia, sete acamados não dispunham deste tipo de superfície de apoio. Assim, foi orientado e demonstrado a importância do colchão para os cuidadores desses sete pacientes. Porém, não foi obtido êxito nesse cuidado prescrito junto aos usuários.

Por outro lado, os cuidados prescritos concernentes ao auxílio na deambulação e a observação no nível de consciência dos acamados, foram atividades em que os cuidadores demonstraram maior envolvimento, por esta razão a efetividade foi de $100 \%$.

Em relação à troca de fraldas foi observado que $50 \%$ dos cuidadores aderiram ao cuidado prescrito, entretanto, os outros $50 \%$ dos cuidadores não aderiram a orientação da troca de fraldas de acordo com as necessidades. Outra evidencia identificada neste estudo foi à baixa ingestão pelos indivíduos investigados de líquidos durante o dia.

No que tange a adesão à prescrição de dieta hipossódica, foi observado que 45,5\% dos cuidadores referiram uma redução de sal no preparo dos alimentos. Quanto à adesão a dieta hipocalórica, dos 11 usuários que possuíam DM, sete seguiram as recomendações relativas à diminuição de carboidratos e gorduras. Neste caso, a adesão de $64 \%$ pode ser atribuída ao receio que os cuidadores tinham de ocorrer piora no estado físico dos pacientes em virtude da diabetes descompensada. 
Entre os indivíduos investigados $25 \%$ seguiram o cuidado de manter a pele seca, apesar do risco aumentado para o desenvolvimento de UP em razão de quase a totalidade dos pacientes mostrarem a pele constantemente úmida ou muito úmida.

Em relação à adesão dos cuidadores a proteção de proeminências ósseas, o estudo demonstrou que dos oito cuidadores orientados para este problema, apenas dois (25\%) aderiram às orientações prescritas. Entretanto, a prescrição para a mudança de decúbito alcançou patamares maiores de adesão, dos oito pacientes que apresentavam problemas em relação a este cuidado, os cuidadores de quatro (50\%) apresentaram atitude positiva a esta recomendação.

\section{Discussões}

$\mathrm{Na}$ amostra estudada a média de idade foi de 80,5 anos $( \pm 4,8)$. Este resultado se aproximou de duas pesquisas com pacientes acamados no domicílio que demonstraram este perfil etário, com médias de idade de 86,7 anos $( \pm 7,6)$ (BRIENZA et al., 2010), e 77,6 ( \pm 11,3) (MORAES et al., 2012). Segundo Chayamiti e Caliri (2010) a idade avançada é apontada como um dos mais relevantes fatores envolvidos na fisiopatogênese da UPP.

Com o avanço da idade, mudanças são observadas na pele, entre elas a diminuição da camada dérmica e de suas propriedades, como a percepção da dor. De acordo com Moraes et al. (2012) a idade avançada favorece a formação de UP em razão da perda de elasticidade da pele, da hidratação cutânea insuficiente e da perda da sensibilidade, dentre outros fatores que ainda podem ser agravados se associados a doenças crônicas, tais como hipertensão arterial sistêmica e Diabetes mellitus, bem como uso de drogas vasoativas que dificultam uma boa perfusão tissular.

Verificou-se que a maioria dos pacientes era pertencente à raça branca, sendo que este resultado está em consonância com os achados de outro estudo, mostrando que a raça branca é mais frequente entre os pacientes acamados (CHAYAMITI; CALIRI,
2010; STUMM et al., 2009). Apesar de este estudo ter encontrado entre os indivíduos investigados idênticas proporções para ambos os sexos, o estudo de Moraes et al. (2012) realizado no domicílio de 40 idosos em Fortaleza e região metropolitana mostrou uma maior prevalência de mulheres entre os idosos acamados.

Aqui é necessário referir que não foram encontradas evidências que associem o aparecimento de UPP em pacientes acamados às variáveis, sexo e raça. De acordo com Nogueira et al. (2012) tais variáveis constituem-se, tão somente em características demográficas.

Evidenciou-se nesta investigação o baixo nível de escolaridade da maioria dos participantes, com predominância $(76 \%)$ do nível fundamental incompleto. A investigação de Chayamiti e Caliri (2010) realizada com a população de pacientes em assistência domiciliária em um Distrito de Saúde de Ribeirão Preto, também revelou baixa escolaridade entre os investigados, entretanto, 40,4\% possuíam $1^{\circ}$ grau incompleto e $29,8 \%$ eram analfabetos. Refletindo, desta forma, o reduzido nível de instrução dos participantes dos dois estudos.

Verificou-se neste estudo uma média elevada do tempo de acamado, próximo de seis anos. Sabese que quando o tempo de acamado se prolonga, aumenta os fatores de intensidade e duração da pressão sobre os tecidos propiciando, desta forma, o desenvolvimento de lesões. Oliveira e Reis (2013) confirmam a partir de um estudo realizado em um hospital público do município de Vitória da Conquista/BA, com 40 idosos portadores de Úlceras de Pressão que a incapacidade de se mover pode afetar a capacidade de aliviar a pressão, predispondo ainda à fricção e às forças de torção se o indivíduo estiver acamado ou ainda confinado à cadeira. De acordo com Luz et al. (2010) a restrição da mobilidade, constitui-se o principal fator intrínseco relacionado à ocorrência de úlcera por pressão.

Observou-se que mais de $70 \%$ dos indivíduos analisados utilizavam o Sistema Único de Saúde (SUS), evidenciando, dessa forma, o importante papel 
que desempenham os serviços de saúde públicos ao responder às necessidades deste tipo de clientela.

Foi possível constatar que a doença de base que preponderava entre os pacientes analisados era o Acidente Vascular Cerebral e que as doenças preexistentes predominantes eram a Hipertensão Arterial e o Diabetes mellitus.

Os estudos descrevem que os pacientes que estão nos domicílios ou participam de programas de reabilitação como aqueles com paraplegia decorrente de lesão medular ou com sequelas de AVC, como população de risco para UPP e que precisam ser avaliados pelos profissionais de saúde, principalmente em razão da diminuição da mobilidade e da sensibilidade. (MORAES et al., 2012; OLIVEIRA et al., 2013). Os diagnósticos de AVC, HÁ, DM e outras doenças crônicas não transmissíveis (DCNTs) podem afetar a capacidade perceptiva, circulação sanguínea, oxigenação, mobilidade, nível de consciência, alterações dos níveis de eletrólitos e proteínas. Ademais podem aumentar a chance de complicações pelo tempo prolongado de permanência do idoso no leito. O estudo de Sales et al. (2010) evidenciou que as doenças neurológicas quando associada à DCNTs, podem afetar a capacidade perceptiva, a circulação sanguínea, a oxigenação e a mobilidade, favorecendo a formação de UPP.

À capacidade de reagir à pressão relacionada ao desconforto, estavamuito limitada ou completamente limitada na metade dos indivíduos estudados. Neste estudo $76 \%$ tinham como doença de base o AVC. Tal situação expõe o paciente ao maior risco para ocorrência de UPP, pois pacientes com alterações neurológicas são mais dependentes de cuidados como mudança de decúbito, higienização íntima a cada evacuação ou micção.

Outro fator importante na gênese das UPs é a exposição da pele à umidade excessiva, provocada pela incontinência urinária, anal e perspiração. No presente estudo, quase que a totalidade (92\%) dos pacientes investigados mostrou a pele constantemente úmida ou muito úmida, sendo que a metade dos indivíduos usava fraldas aumentando, portanto, a possibilidade da presença de umidade excessiva na pele. Segundo Chayamiti e Caliri (2010), a exposição excessiva a umidade, acarreta maceração e enfraquecimento das camadas superficiais da pele. De acordo com Zambonato, Assis e Beghetto (2013) ter a subescala umidade da Escala de Braden alterada aumenta em quatro vezes o risco de pacientes acamados de desenvolver a UPP, o que corrobora o seu caráter preditivo a esse tipo de lesão.

Em relação à mobilidade, quase a totalidade dos pacientes estudados apresentaram-se completamente ou muito limitados. A mobilidade prejudicada aliada à restrição ao leito e a cadeira pode ser considerada como um dos principais fatores responsáveis pelo desenvolvimento de úlceras de pressão. Entretanto, apesar do problema, $58 \%$ dos participantes do estudo não utilizavam superfícies de apoio como, por exemplo, colchões piramidais. O estudo de Brienza et al. (2010) com pacientes acamados comprova que o uso de almofadas ou colchões que reduzem pressões perto das proeminências ósseas, que regulam a dissipação de calor e umidade é uma medida eficiente na prevenção de úlcera por pressão.

Foi constatado que nenhum paciente estudado apresentou nutrição adequada. O estado nutricional das pessoas acamadas geralmente está comprometido, devido a fatores como desnutrição e estados patológicos. Os problemas nutricionais, a curto e em longo prazo, podem predispor os pacientes à formação da úlcera por pressão (PEDRONI; BONATTO; MENDES, 2014). O estado nutricional ruim é apontado por Sales et al. (2010) como um determinante na formação da UPP por contribuir para a diminuição da tolerância tissular à pressão e por retardar o processo de cicatrização. Vale lembrar que a avaliação do estado nutricional pela Escala de Braden, possui limitações, pois ela avalia a ingestão e não o Índice de Massa Corporal, apetite e história alimentar (BRADEN; BERGSTROM, 1987). 
A baixa ingesta hídrica esteve presente para todos os participantes, e a média encontrada por individuo foi de $475 \mathrm{ml} /$ dia. A necessidade hídrica diária do adulto está entre 1500 e $2400 \mathrm{ml} /$ dia (FERREIRA, 2011). A baixa oferta de substancia líquida aliada a uma nutrição inadequada torna-se um importante fator de risco para UPP por reduzir a elasticidade da pele. O estudo de Lobo (2008) demonstra a existência de correlação estatística entre o aumento da ingesta hídrica e a diminuição no risco de desenvolver úlcera por pressão.

Em relação à análise dos fatores de risco para UPP, considerando-se os escores de cada uma das subescalas (percepção sensorial, umidade, atividade, mobilidade, nutrição, fricção e cisalhamento), observou-se que as subescalas que obtiveram menores escores nesta pesquisa, e que mais contribuem para a gravidade do risco para o desenvolvimento de UPP foram à umidade da pele, mobilidade, fricção e cisalhamento. Estes dados são corroborados em estudo semelhante realizado em hospitais brasileiros (OLIVEIRA; REIS, 2013; ZAMBONATO; ASSIS; BEGHETTO, 2013).

Gomes et al. (2011) ao analisar os fatores de risco para o desenvolvimento de úlcera por pressão em pacientes adultos internados em Centros de Terapia Intensiva, em Belo Horizonte, identificou que os pacientes acamados que apresentavam escore hum para percepção sensorial, hum ou dois para umidade da pele, hum para atividade física, hum para mobilidade, dois para nutrição e hum fricção para cisalhamento, foram os que tiveram o maior número de úlceras por pressão.

Ao avaliar a adesão dos cuidadores aos cuidados propostos na prevenção de UPP, foi observado que houve uma boa adesão, interesse e envolvimento dos cuidadores para as ações, inspecionar e hidratar a pele, oferecer dieta pastosa, deixar objetos ao alcance do paciente, auxiliar na deambulação, observar o nível de consciência do acamado e oferecer dieta hipocalórica, para aqueles que possuíam DM. As ações com menor adesão foram referentes a aumentar a ingesta hídrica, utilizar o colchão piramidal, ofertar dieta hipossódica aos hipertensos e proteger as proeminências ósseas.

Neste estudo 10 tinham problemas com relação à ingesta hídrica. Apesar disto, os resultados referentes ao aumento da oferta hídrica foram de 30\%. Em relação aos outros dois usuários, constatou-se que eles ingeriam em torno de $1500 \mathrm{ml} /$ dia de líquido através da gastrostomia, valor que de acordo com a escala de Braden é suficiente para pessoas acamadas (CONCEIÇÃO, 2010).

Em relação ao cuidado prescrito da troca de fraldas na presença de umidade, a adesão foi parcial. Visto que a metade dos cuidadores realizou a troca de fralda de maneira mais frequente, evitando que a umidade permanecesse em contato com a pele do usuário acamado. Entretanto, a outra metade dos cuidadores não aderiu à orientação da troca de fraldas de acordo com as necessidades, pois mantiveram a exposição excessiva da pele do paciente a umidade. Conferindo, portanto, aos pacientes risco para o desenvolvimento de UP.

Chama a atenção o fato de que os cuidadores não seguiram a prescrição relativa ao uso de colchão piramidal. Brienza et al. (2010) têm referido que a resistência em pacientes com baixa mobilidade e imersão inadequada nas superfícies almofadas e colchões pode conduzir a uma pressão de indução de isquemia prolongada e de cisalhamento que se acredita ser o fator primário que conduz ao desenvolvimento de UPP.

Estudos evidenciam que as comorbidades como hipertensão e doenças cardiovasculares que comprometem a perfusão tecidual são significativas para o desenvolvimento das UPP (MENEGON et al., 2012; MORAES et al., 2012; SALES; BORGES; DANOSO, 2010). Neste estudo a adesão à dieta hipossódica obteve resultado inferior àquele observado por Cunha et al. (2012) em um estudo com hipertensos no município de São Luís/MA, onde o percentual na redução do sal foi de 72,7\%. No caso dos outros pacientes desta investigação 
foi constatado que os cuidadores, ora ofereciam a comida que toda a família ingeria, ora preparavam as sopas sem o cuidado de colocar pouco sal.

Tanto a mudança de decúbito, quanto a proteção das proeminências ósseas estão descritas na literatura como intervenções necessárias para a prevenção de UPP em pessoas acamadas (BRIENZA et al., 2010). Apesar dos cuidadores terem recebido orientações de como controlar o excesso de pressão nas proeminências ósseas dos acamados investigados, este tipo de cuidado não recebeu a devida importância, em razão da baixa adesão (25\%) na proteção dessas proeminências. Entretanto, na mudança de decúbito foi percebido um maior interesse dos cuidadores, pois 50\% colaboraram realizando esse cuidado.

A úlcera por pressão ocorre quando a pressão intersticial excede a pressão intracapilar, originando uma deficiência de perfusão capilar, o que impede o transporte de nutrientes ao tecido, ocasionando a isquemia. Esta situação é mais comum em áreas de proeminências ósseas, onde o osso e a superfície de contato a qual o paciente se apoia. Quando esta isquemia tecidual gerada pela pressão é mantida por um tempo maior do que o necessário à recuperação do tecido frente à isquemia, ocorre a liberação de fatores inflamatórios. Os fatores inflamatórios alteram a permeabilidade vascular, gerando edema e piorando a isquemia, caso a pressão intersticial permaneça aumentada. A isquemia a nível celular leva à morte celular, gerando a liberação de mais fatores inflamatórios e fatores de necrose tecidual (WADA; TEIXEIRA; FERREIRA, 2010). O estudo de Wada, Teixeira e Ferreira (2010) refere que o alívio da pressão pela mudança de decúbito sobre uma proeminência óssea por cinco minutos a cada duas horas permite a adequada recuperação do tecido à agressão isquêmica e evita, muitas vezes, a formação da lesão.

Apesar da reconhecida importância de se conhecer as razões que levam os pacientes e cuidadores a não aderirem aos cuidados prescritos, este estudo mostrou limitações, pois não avaliou quais os motivos que contribuíram para a não aderência no uso do colchão piramidal e na baixa aderência à proteção das proeminências ósseas, ao aumento da ingesta hídrica, a oferta de dieta hipossódica e ao cuidado com a pele.

\section{Conclusões}

Em conclusão, os achados encontram-se em consonância com outros estudos, pois os dados mostram as semelhanças entre o perfil sociodemográfico e as condições clínicas dos indivíduos acamados deste estudo com os dados de indivíduos estudados e descritos na literatura.

Conhecer a vulnerabilidade das pessoas acamadas no domicílio ao risco de desenvolver úlcera por pressão pode permitir aos profissionais das Unidades Básicas de Saúde identificar os indivíduos mais susceptíveis e implantar ou intensificar as medidas preventivas necessárias.

Outro aspecto constatado neste estudo foi a adesão parcial à prescrição reforçando, desta forma, a importância de aprofundar a compreensão das dificuldades inerentes ao processo de adesão às prescrições dos cuidados na prevenção de UP, a fim de melhorar a assistência aos pacientes acamados.

Para finalizar, este estudo reitera a relevância da utilização da escala de Braden na assistência em saúde como instrumento eficiente na predição de risco para o desenvolvimento de UPP em pacientes acamados no domicílio.

\section{Referências}

ARAÚJO, T. M.; MOREIRA, M. P.; CAETANO, J. A. Avaliação de risco para úlcera por pressão em pacientes críticos. Revista Enfermagem, Rio de Janeiro, v. 19, n. 1, p. 58-63, 2011. Disponível em: $<$ http://www.facenf.uerj.br/v19n1/v19n1a10.pdf $>$. Acesso em: 29 jun. 2015.

BRADEN, B.; BERGSTROM, N. A conceptual schema for the study of the etiology of pressure sores. Rehabilitation Nursing, Chicago, v. 12, n. 1, p. 8-12, 1987. 
BRIENZA， D.; KELSEY， S.; KARG, P.; ALLEGRETTI, A.; OLSON, M.; SCHMELER, M.; ZANCA, J.; GEYER, M. J.; KUSTURISS, M.; HOLM, M. A Randomized clinical trial on preventing pressure ulcers with wheelchair seat cushions. Journal of the American Geriatrics Society, Pittsburgh, v. 58, n. 12, p. 2308-2314, 2010. Disponível em: <http:// www.redalyc.org/pdf/4038/403838775003.pdf $>$. Acesso em: 23 out. 2015.

BRITO, K. K. G.; SOARES, M. J. G. O.; SILVA, M. A. Cuidado de enfermagem nas ações preventivas nas úlceras de pressão. Revista Brasileira de Ciências da Saúde, João Pessoa, n. ano 12, n. 40, p. 56-61, abr./jun. 2014. Disponível em: $\quad<$ http://www.ufrgs.br/educatele/cursos/ arquivos/cuidado-de-enfermagem-nas-acoespreventivas-nas-ulceras-de-pressao $>$. Acesso em: 9 jan. 2016.

CHAYAMITI, E. M. P. C.; CALIRI, M. H. L. Úlcera por pressão em pacientes sob assistência domiciliária. Acta Paulista de Enfermagem, São Paulo, v. 23, n. 1, p. 29-34, 2010. Disponível em: $<$ http://www.scielo.br/pdf/ape/v23n1/05.pdf $>$. Acesso em: 19 maio 2015.

CONCEIÇÃO, L. F. S. Saúde do idoso: orientações ao cuidador do idoso acamado. Revista Médica de Minas Gerais, Belo Horizonte, v. 20, n. 1, p. 81-91, 2010. Disponível em: <http://www. observatorionacionaldoidoso.fiocruz.br/biblioteca/ artigos/199.pdf $>$. Acesso em: 14 out. 2015.

CUNHA, P. R. M. S.; BRANCO, D. R. C.; BERNARDES, A. C. F.; AGUIAR, M. I. F.; ROLIM, I. L. T. P.; LINARD, A. G. Prevalência e causas de não adesão ao tratamento antihipertensivo de idosos na atenção básica. Revista de Pesquisa em Saúde, São Luiz, v. 13, n. 3, p. 11-16, 2012. Disponível em: <http://www.ebserh. gov.br/documents/16424/491465/Revista13-3 set-dez-2012.pdf/045c3b26-4c7c-4965-83753ae96e632553>. Acesso em: 9 jan. 2016.

FERREIRA, L. G. B. Terapia de hidratação venosa. Revista Hospital Universitário Pedro Ernesto, Rio de Janeiro, v. 10, s. 2, 2011. Disponível em: $<$ http://revista.hupe.uerj.br/default.asp?ed=23>. Acesso em: 8 jan. 2016.
GOMES, F. S.L.;BASTOS, M.A. R.; MATOZINHOS, F. P.; TEMPONI, H. R.; VELASQUEZ-MELÉNDEZ, G. Avaliação de risco para úlcera por pressão em pacientes críticos. Revista da Escola de Enfermagem da USP, São Paulo, v. 45, n. 2, p. 313-318, 2011. Disponível em: <http://dx.doi.org/10.1590/S0080$62342011000200002>$. Acesso em: 27 out. 2015.

LUZ, R. P.; LOPACINSKI, A. C.; FRAGA, R.; URBAN, C. A. Úlceras de pressão. Revista de Geriatria \& Gerontologia, Rio de janeiro, v. 4, p. 36-43, 2010. Disponível em: <http://sbgg.org.br/publicacoescientificas/revista-geriatria-gerontologia/ $>$. Acesso em: 11 nov. 2015.

MENEGON, D. B.; BERCINI, R. R.; SANTOS, C. T.; LUCENA, A. F.; PEREIRA, A. G. S.; SCAIN, S. F. Análise das subescalas de Braden como indicativos de risco para úlcera por pressão. Texto \& Contexto: Enfermagem, Florianópolis, v. 21, n. 4, p. 854-861, 2012. Disponível em: <http://dx.doi. org/10.1590/S0104-07072012000400016>. Acesso em: 8 jan. 2016.

MORAES, G. L. A.; ARAUJO, T. M.; CAETANO, J. A.; LOPES, M. V. O.; SILVA, M. J. Avaliação de risco para úlcera por pressão em idosos acamados no domicílio. Acta Paulista de Enfermagem, São Paulo, v. 25, n. 1, p. 7-12, 2012.

OLIVEIRA, A. R. S.; COSTA, A. G. S.; ARAUJO, T. L.; AQUINO, P. S.; PINHEIRO, A. K. B.; XIMENES, L. B. Competências essenciais de programas de assistência domiciliar para pacientes com acidente vascular cerebral. Revista Eletrônica de Enfermagem, Goiânia, v. 15, n. 2, p. 317-325, 2013. Disponível em: $<$ http://dx.doi.org/10.5216/ree.v15i2.18046>. Acesso em: 22 out. 2015.

OLIVEIRA, N.; REIS, L. A. Caracterização das úlceras por pressão em idosos hospitalizados. Revista Enfermagem Contemporânea, Salvador, v. 2, n. 1, p. 146-156, 2013.

PEDRONI, L.; BONATTO, S.; MENDES, K. O impacto da desnutrição no desenvolvimento e na gravidade das úlceras por pressão: uma revisão da literatura. Revista Brasileira de Ciências do Envelhecimento Humano, Passo Fundo, v. 11, n. 1, p.89-102, 2014. Disponível em: <http://www.upf. br/seer/index.php/rbceh/article/view/3891/pdf>. Acesso em: 22 out. 2015. 
PESSOA, E. F. R.; ROCHA, J. G. S. C.; BEZERRA, S. M. G. Prevalência de úlcera por pressão em pacientes acamados, cadastrados na Estratégia de Saúde da Família: um estudo de enfermagem. Revista Interdisciplinar NOVAFAPI, Teresina, v. 4, n. 1, p. 1418, 2011. Disponível em: <http://www.novafapi.com. $\mathrm{br} /$ sistemas/revistainterdisciplinar/v4n1/pesquisa/p2_ v4n1.pdf. $>$. Acesso em: 7 jan. 2016.

POSS, J.; MURPHY, M.K.; WOODBURY, M.G.; ORSTED, H.; STEVENSON, K.; MACALPINE,S.; CURTIN-TELEGDI, N.;HIRDES, J.P. Development of the interRAI Pressure Ulcer Risk Scale (PURS) for use in long-term care and home care settings. BMC Geriatrcs, London, v. 20, n. 10, p. 67, 2010. Disponível em: <http://www.biomedcentral.com/ content/pdf/1471-2318-10-67.pdf.>. Acesso em: 20 jun. 2015.

PREFEITURA MUNICIPAL DE JOINVILLE. Fundação Instituto de Pesquisa e Planejamento para o Desenvolvimento Sustentável de Joinville. Joinville bairro a bairro. 2015. Disponível em: $<$ https://ippuj.joinville.sc.gov.br/arquivo/lista/ codigo/598-Vers\%C3\%A3o\%2B2015.html>. Acesso em: 20 jun. 2015.

ROCHA FILHO, D. R.; OLIVEIRA, D. C.; CARVALHO, A. R. B.; LEAL, M. G. Produção científica sobre as abordagens preventivas das úlceras por pressão. Revista Interdisciplinar, São Luís, v. 6, n. 4, p. 196-204, 2013. Disponível em: http://revistainterdisciplinar.uninovafapi.edu.br/ index.php/revinter/article/viewFile/227/pdf_8. Acesso em: 07 jan. 2016.

SALES, M. C. M.; BORGES, E. L.; DANOSO, M. T. V. Risco e prevalência de úlceras por pressão em uma unidade de internação de um hospital universitário de Belo Horizonte. Revista Mineira de Enfermagem, Belo Horizonte, v. 14, n. 4, p. 566575, 2010. Disponível em: <http://www.reme.org. br/artigo/detalhes/152>. Acesso em: 8 jan. 2016.

STUMM, E. M. F.; ZAMBONATO, D.; KIRCHNER, R. M.; DALLEPIANE, L. B.; BERLEZI, E. M. Perfil de idosos assistidos por unidades de Estratégia de Saúde da Família que sofreram infarto agudo do miocárdio. Revista Brasileira de Geriatria e Gerontologia, São Paulo, v. 12, n. 3, p. 449-461, 2009. Disponível em: <http://www.redalyc.org/articulo. oa? $\mathrm{id}=403838782011>$. Acesso em: 22 out. 2015.
WADA, A.; TEIXEIRA, N. N.; FERREIRA, M. C. Úlceras por pressão. Revista Médica, São Paulo, v. 89, n. 3/4, p. 170-177, 2012. Disponível em: $<$ http:// scholar.google.com.br/scholar_url?url=http://www. revistas.usp.br/revistadc/article/download/46293/499 $49 \& \mathrm{hl}=\mathrm{ptBR} \& \mathrm{sa}=\mathrm{X} \& \mathrm{scisig}=\mathrm{AAGBfm} 219 \mathrm{vMzXs} 4$ Ha6tQqS11eQf_dN5cDQ\&nossl=1\&oi=scholarr\&ve $\mathrm{d}=0 \mathrm{CBwQgAMoADAAahUKEwjFpP2btDIAhUL1J}$ AKHfPuBS0>. Acesso em: 11 jun. 2015.

ZAMBONATO, B. P.; ASSIS, M. C. S.; BEGHETTO, M. G. Associação das sub-escalas de Braden com o risco do desenvolvimento de úlcera por pressão. Revista Gaúcha de Enfermagem, Porto Alegre, v. 34, n. 1, p. 21-28, 2013. Disponível em: $<$ http://www.scielo.br/pdf/rgenf/v34n2/v34n2a03. pdf $>$. Acesso em: 8 jan. 2016. 